\title{
薄膜结构及形貌对钙钛矿电池性能的影响
}

\author{
郡志鹏 ${ }^{a}$ 潘旭 ${ }^{*, a} \quad$ 张旭辉 $^{a}$ 叶加久 ${ }^{a}$ 朱梁正 ${ }^{a}$ 李毅 ${ }^{a}$ \\ 马艳梅 ${ }^{a} \quad$ 黄阳 $^{a} \quad$ 朱俊 $^{a} \quad$ 胡林华 ${ }^{a} \quad$ 孔太 ${ }^{a} \quad$ 戴松元 ${ }^{*, a, b}$ \\ $\left({ }^{a}\right.$ 中国科学院等离子体物理研究所 新型薄膜太阳电池重点实验室 合肥 230031) \\ $(b$ 华北电力大学 新型薄膜太阳电池北京市重点实验室 北京 102206)
}

\begin{abstract}
摘要 采用液相连续沉积法制备了有机/无机杂化钻铁矿太阳电池, 并研究了不同形貌钙钛矿 $\left(\mathrm{CH}_{3} \mathrm{NH}_{3} \mathrm{PbI}_{3}\right)$ 光活性层对 太阳电池性能的影响. 实验结果表明在连续沉积法中 $\mathrm{PbI}_{2}$ 的结构对 $\mathrm{CH}_{3} \mathrm{NH}_{3} \mathrm{PbI}_{3}$ 层的形貌具有重要的影响, $\mathrm{PbI}_{2}$ 薄膜中 适当的孔洞结构有利于其与 $\mathrm{CH}_{3} \mathrm{NH}_{3} \mathrm{I}$ 充分反应形成 $\mathrm{CH}_{3} \mathrm{NH}_{3} \mathrm{PbI}_{3}$ 层. 致密的 $\mathrm{PbI}_{2}$ 层造成 $\mathrm{PbI}_{2}$ 的转化不完全, 导致 $\mathrm{CH}_{3} \mathrm{NH}_{3} \mathrm{PbI}_{3}$ 颗粒较小, 吸收较弱, 影响电池的短路电流. 而 $\mathrm{CH}_{3} \mathrm{NH}_{3} \mathrm{PbI}_{3}$ 颗粒过大会引起 $\mathrm{CH}_{3} \mathrm{NH}_{3} \mathrm{PbI}_{3}$ 薄膜孔洞产生, 造成电池的开路电压下降. 通过对电池制备工艺的优化获得了 $13.5 \%$ 的最佳光电转换效率.
\end{abstract}

关键词 有机/无机杂化; 钻铁矿; 制备工艺; 形貌; 太阳电池

\section{Influence of Structure and Morphology of Perovskite Films on the Performance of Perovskite Solar Cells}

\author{
Shao, Zhipeng ${ }^{a} \quad{\text { Pan, } \mathrm{Xu}^{*, a} \quad \text { Zhang, Xuhui }^{a} \quad \text { Ye, Jiajiu }^{a} \quad \text { Zhu, Liangzheng }}^{a}$

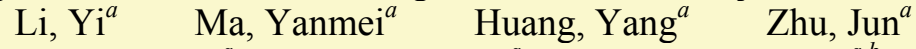 \\ $\mathrm{Hu}$, Linhua $^{a}$ Kong, Fantai $^{a}$ Dai, Songyuan*,a,b \\ ( ${ }^{a}$ Key Laboratory of Novel Thin Film Solar Cells, Institute of Plasma Physics, Chinese Academy of Sciences, \\ Hefei, 230031) \\ $\left({ }^{b}\right.$ Beijing Key Laboratory of Novel Thin Film Solar Cells, North China Electric Power University, Beijing, 102206)
}

\begin{abstract}
Perovskite solar cells based on the inorganic/organic hybrid perovskite have attracted increasing attention over the past 3 years. Many studies have been done in this area. Controling the morphology of the perovskite film is an effective way to improve the photoelectric conversion efficiency of the devices. In our reserch, we studied the influence of structure and morphology of perovskite films on the performance of the organic-inorganic hybrid perovskite solar cells which prepared by a sequential deposition method. Mesoporous $\mathrm{TiO}_{2}$ scaffold were introduced as electron collecting layer. Lead iodide $\left(\mathrm{PbI}_{2}\right)$ was then spin cast on the $\mathrm{TiO}_{2}$ scaffold. The $\mathrm{PbI}_{2}$ subsequently transformed into the perovskite $\left(\mathrm{CH}_{3} \mathrm{NH}_{3} \mathrm{PbI}_{3}\right)$ by dipping the $\mathrm{TiO}_{2} / \mathrm{PbI}_{2}$ film into a solution of $\mathrm{CH}_{3} \mathrm{NH}_{3} \mathrm{I}$. We studied the difference between the $\mathrm{PbI}_{2}$ film with or without drying under room temperature after spin-coating. Through drying under room temperature, larger pores formed in the $\mathrm{PbI}_{2}$ film. $\mathrm{While}$ without drying under room temperature, smaller and shallower pores formed in the $\mathrm{PbI}_{2}$ film. The results show that larger pores in $\mathrm{PbI}_{2}$ film leads to more complete transformation of $\mathrm{PbI}_{2}$ to $\mathrm{CH}_{3} \mathrm{NH}_{3} \mathrm{PbI}_{3}$ and larger $\mathrm{CH}_{3} \mathrm{NH}_{3} \mathrm{PbI}_{3}$ particles. $\mathrm{CH}_{3} \mathrm{NH}_{3} \mathrm{PbI}_{3}$ films were prepared with three different processes: (a) direct dipping the $\mathrm{PbI}_{2}$ film with smaller pores into the $\mathrm{CH}_{3} \mathrm{NH}_{3} \mathrm{I}$ solution; (b) direct dipping the $\mathrm{PbI}_{2}$ with larger pores into the $\mathrm{CH}_{3} \mathrm{NH}_{3} \mathrm{I}$ solution; (c) dipping the $\mathrm{PbI}_{2}$ with larger pores into the $\mathrm{CH}_{3} \mathrm{NH}_{3} \mathrm{I}$ solution after pre-wetting. The resulting $\mathrm{CH}_{3} \mathrm{NH}_{3} \mathrm{PbI}_{3}$ films were studied with SEM, UV-vis absorption spectrum and XRD. The particles size of the $\mathrm{CH}_{3} \mathrm{NH}_{3} \mathrm{PbI}_{3}$ are 150, 250 and $350 \mathrm{~nm}$ for process (a), (b) and (c) respectively. $\mathrm{CH}_{3} \mathrm{NH}_{3} \mathrm{PbI}_{3}$ films fabricated through process (a) show insufficient absorption due to the insufficient transformation of the $\mathrm{PbI}_{2}$. The pre-wetting procedure leads to slower reaction result in larger $\mathrm{CH}_{3} \mathrm{NH}_{3} \mathrm{PbI}_{3}$ particle size. Devices with proper size of $\mathrm{CH}_{3} \mathrm{NH}_{3} \mathrm{PbI}_{3}$ particles show the highest photoelectric conversion efficiency. An efficiency of $13.5 \%$ was achieved with a $J_{\mathrm{sc}}$ of $17.8 \mathrm{~mA} / \mathrm{cm}^{2}$, a $V_{\mathrm{oc}}$ of $1.05 \mathrm{~V}$ and a $\mathrm{FF}$ of $72.5 \%$.

Keywords organic/inorganic hybrid; perovskite; preparation process; morphology; solar cell
\end{abstract}

\section{1 引言}

有机/无机杂化钙钛矿太阳电池作为太阳电池中一
个新兴的分支在近几年获得了迅速的发展 ${ }^{[1]}$. 自从 2009 年 Miyasaka 研究组首次利用有机/无机钻钛矿材料作为 敏化剂成功制备了钙钛矿敏化太阳电池，并获得了

*E-mail: sydai@ipp.ac.cn; mars_dark@hotmail.com

Received October 17, 2014; published December 25, 2014

Project supported by the National Basic Research Program of China (No. 2011CBA00700), the National High Technology Research and Development Program of China (No. 2011AA050510), and the National Natural Science Foundation of China (Nos. 21103197, 21273242).

项目受国家 973 计划(No. 2011CBA00700)、国家 863 计划(No. 2011AA050510)和国家自然科学基金(Nos. 21103197, 21273242)资助. 
$3.8 \%$ 的光电转换效率. 在最近五年中有机/无机钙钛矿 电池光电转换效率取得了飞速的提高 ${ }^{[2]}$. 目前钲钛矿电 池的实验室效率已达到 $19.3 \%$, 已接近目前商业化的太 阳电池光电转换效率 ${ }^{[3]}$. 相比于传统的太阳电池有机/无 机钙钛矿太阳电池更具有制备工艺简单, 低能耗, 低成 本的优点, 有望取代传统的硅基太阳电池 ${ }^{[4]}$.

有机/无机钙钛矿半导体材料种类多, 带隙可调, 适 合用于太阳电池中 ${ }^{[5]}$. $\mathrm{CH}_{3} \mathrm{NH}_{3} \mathrm{PbI}_{3}$ 是目前在太阳电池中 研究最多的有机/无机半导体材料 ${ }^{[6]} . \mathrm{CH}_{3} \mathrm{NH}_{3} \mathrm{PbI}_{3}$ 是一 种直接带隙导体材料, 带隙宽度为 $1.55 \mathrm{eV}$, 在可见光区 域具有高摩尔消光系数 $\left(10^{5} \mathrm{~cm}^{-1}\right)$, 较宽的光谱吸收范 围, 吸收光谱覆盖整个可见光区域, 并且具有优异的载 流子传输性能, 其电子空穴扩散距离达到 $100 \mathrm{~nm}$, 空穴 扩散距离达到 $1000 \mathrm{~nm}^{[7]}$. $\mathrm{CH}_{3} \mathrm{NH}_{3} \mathrm{PbI}_{3}$ 在极性溶剂如 DMF 和 $\gamma$-丁内酯中具有良好的溶解性能易于溶液法制 备, 制备工艺简便 ${ }^{[1 \mathrm{a}, 8]}$.

基于有机/无机钙钛矿材料的太阳电池一般有两种 结构: 一种是含有多孔骨架层作为电子或空穴传输材料 的钙钛矿敏化电池结构, 另一种是不含多孔骨架层的平 板异质结结构 ${ }^{[9]}$. 多孔骨架结构电池通常采用在导电玻 璃上制备一层致密空穴阻挡层, 并在致密阻挡层上制备 $\mathrm{TiO}_{2}$ 多孔骨架结构作为电子传输层, 然后在 $\mathrm{TiO}_{2}$ 多孔 骨架结构上制备一层 $\mathrm{CH}_{3} \mathrm{NH}_{3} \mathrm{PbI}_{3}$ 光活性层, 最后在光 活性层上旋涂一层空穴传输层, 空穴传输层通常选用 2,2',7,7'-四[ $N, N$-二(4-甲氧基苯基)氨基]-9,9'-螺二芴 (Spiro-OMeTAD) ${ }^{[10]}$. 基于这种结构的太阳电池已取得 $17 \%$ 的实验室光电转换效率 ${ }^{[11]}$. 由于 $\mathrm{CH}_{3} \mathrm{NH}_{3} \mathrm{PbI}_{3}$ 具有 优异的电子空穴传输性能, 绝缘的 $\mathrm{Al}_{2} \mathrm{O}_{3}$ 多孔膜同样被 应用于制备骨架层 ${ }^{[9 a, 12]}$. 基于 $\mathrm{Al}_{2} \mathrm{O}_{3}$ 骨架层的电池也取 得了超过 $15 \%$ 的光电转换效率 ${ }^{[13]}$. 利用具有 $\mathrm{p}$ 型导电能 力的 $\mathrm{NiO}$ 制备骨架结构的反向太阳电池同样取得了超 过 $10 \%$ 的光电转换效率 ${ }^{[14]}$. 平板结构电池通常采用 $\mathrm{TiO}_{2}, \mathrm{PCBM}$ 或 $\mathrm{ZnO}$ 等作为电子传输层, 利用 SpiroOMeTAD, PEDOT: PSS, PTAA 等制备空穴传输层 ${ }^{[15]}$. 基于平板结构的有机/无机钻钛矿电池目前已经达到 $19.3 \%$ 的光电转换效率 ${ }^{[3]}$.

目前研究人员开发了多种 $\mathrm{CH}_{3} \mathrm{NH}_{3} \mathrm{PbI}_{3}$ 光活性层制 备方法, 包括: 旋涂法, 双源共蒸发法, 连续沉积法以 及蒸汽辅助溶液法等 ${ }^{[9 b, 16]}$. 研究发现不同的制备工艺对 $\mathrm{CH}_{3} \mathrm{NH}_{3} \mathrm{PbI}_{3}$ 光活性层的形貌以及覆盖率具有很大影响, 从而对电池的光电转换性能产生重要影响 ${ }^{[12,17]}$. 本文中 我们针对连续沉积法研究了不同的制备工艺引起的 $\mathrm{CH}_{3} \mathrm{NH}_{3} \mathrm{PbI}_{3}$ 光活性层薄膜的形貌的不同对太阳电池光 电转换性能的影响. 通过对研究工艺的优化获得了 $13.5 \%$ 的光电转换效率.

\section{2 结果与讨论}

\section{1 有机/无机钙钛矿电池结构}

采用两步连续沉积法制备的有机/无机钙钛矿太阳 电池结构如图 1 所示. 首先在 FTO 玻璃上制备一层致密 的 $\mathrm{TiO}_{2}$ 致密层, 致密层的厚度为 $20 \mathrm{~nm}$. 然后在致密层 上制备一层 $350 \mathrm{~nm}$ 的 $\mathrm{TiO}_{2}$ 多孔骨架层. 在骨架层上制 备一层 $\mathrm{PbI}_{2}, \mathrm{PbI}_{2}$ 渗入 $\mathrm{TiO}_{2}$ 多孔结构中, 并且在 $\mathrm{TiO}_{2}$ 骨 架层表面形成一层覆盖层. 然后通过 $\mathrm{PbI}_{2}$ 与 $\mathrm{CH}_{3} \mathrm{NH}_{3} \mathrm{I}$ 反应生成 $\mathrm{CH}_{3} \mathrm{NH}_{3} \mathrm{PbI}_{3}$ 光活性层, 活性层覆盖层厚度为 $250 \mathrm{~nm}$. 然后, 在 $\mathrm{CH}_{3} \mathrm{NH}_{3} \mathrm{PbI}_{3}$ 光活性层上制备一层 Spiro-OMeTAD 作为空穴传输材料. 最后再蒸镀一层金 电极.

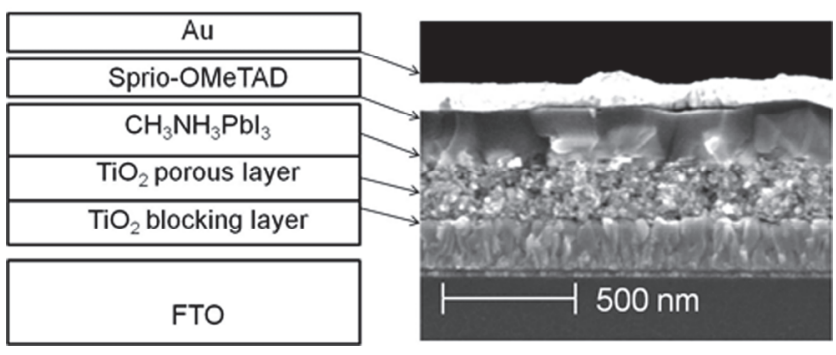

图 1 有机/无机钻钛矿太阳电池结构示意图与断面 SEM 图.

Figure 1 Diagrammatic sketch and SEM image of the organic-inorganic hybrid perovskite solar cells.

\section{2 不同方法制备 $\mathrm{Pbl}_{2}$ 薄膜}

本研究中我们采用两种方法制备 $\mathrm{PbI}_{2}$ 层. 可以看 出, 在空气中缓慢晾干过程对 $\mathrm{PbI}_{2}$ 薄膜形貌产生很大影 响. 图 2 比较了 $\mathrm{PbI}_{2}$ 旋涂后直接 $70{ }^{\circ} \mathrm{C}$ 烘干(a)以及旋涂 后在室温下晾干后 $70{ }^{\circ} \mathrm{C}$ 烘干(b)对 $\mathrm{PbI}_{2}$ 薄膜形貌的影 响. 旋涂之后直接烘干获得的 $\mathrm{PbI}_{2}$ 薄膜较致密. 在空气 中晾干使得 $\mathrm{PbI}_{2}$ 颗粒变大, 并且 $\mathrm{PbI}_{2}$ 薄膜之间空隙变 大，这使得在下一步反应生成 $\mathrm{CH}_{3} \mathrm{NH}_{3} \mathrm{PbI}_{3}$ 光活性层过 程中 $\mathrm{CH}_{3} \mathrm{NH}_{3} \mathrm{I}$ 的异丙醇溶液更容易渗入到 $\mathrm{PbI}_{2}$ 薄膜的 空隙当中, 有利于 $\mathrm{CH}_{3} \mathrm{NH}_{3} \mathrm{PbI}_{3}$ 的生成以及 $\mathrm{CH}_{3} \mathrm{NH}_{3} \mathrm{PbI}_{3}$ 晶粒的长大.

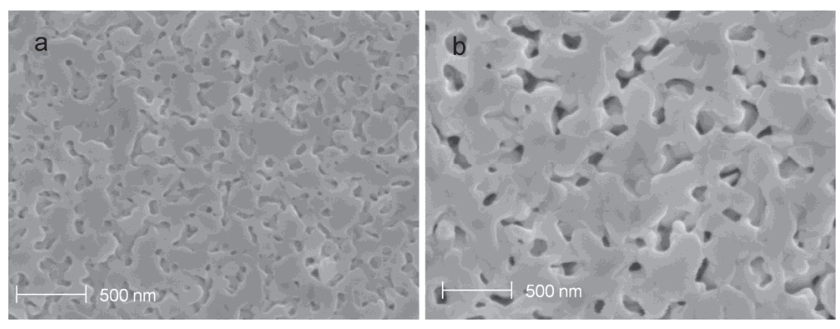

图 2 不同方法制备 $\mathrm{PbI}_{2}$ 薄膜的 $\mathrm{SEM}$ 图. (a) $\mathrm{PbI}_{2}$ 旋涂后直接 $70{ }^{\circ} \mathrm{C}$ 烘 干, (b) $\mathrm{PbI}_{2}$ 旋涂后在室温下晾干后 $70{ }^{\circ} \mathrm{C}$ 烘干.

Figure 2 SEM images of $\mathrm{PbI}_{2}$ with different fabrication methods. (a) without drying at room temperature; (b) drying at room temperature after spincoating. 


\section{3 不同方法制备 $\mathrm{CH}_{3} \mathrm{NH}_{3} \mathrm{Pbl}_{3}$ 薄膜}

本研究中利用三种不同的方法制备 $\mathrm{CH}_{3} \mathrm{NH}_{3} \mathrm{PbI}_{3}$ 光 活性层. (a) 将 $\mathrm{PbI}_{2}$ 溶液旋涂在 $\mathrm{TiO}_{2}$ 薄膜上, 旋涂之后立 即放置在 $70{ }^{\circ} \mathrm{C}$ 的加热台上退火 $30 \mathrm{~min}$. 冷却后浸入 $\mathrm{CH}_{3} \mathrm{NH}_{3} \mathrm{I}$ 的异丙醇溶液中 $20 \mathrm{~s}$, 取出旋干, $70{ }^{\circ} \mathrm{C}$ 下退火 30 min. (b) 将 $\mathrm{PbI}_{2}$ 溶液旋涂在 $\mathrm{TiO}_{2}$ 薄膜上, 旋涂之后在 室温下晾干, 之后放置在 $70{ }^{\circ} \mathrm{C}$ 的加热台上退火 $30 \mathrm{~min}$. 冷却后浸入 $\mathrm{CH}_{3} \mathrm{NH}_{3} \mathrm{I}$ 的异丙醇溶液中 $20 \mathrm{~s}$, 取出旋干, 在 $70{ }^{\circ} \mathrm{C}$ 下退火 $30 \mathrm{~min}$. (c) 将 $\mathrm{PbI}_{2}$ 溶液旋涂在 $\mathrm{TiO}_{2}$ 薄膜 上, 室温下晾干, 然后在异丙醇溶液中浸润 $2 \mathrm{~s}$, 接着浸 入 $\mathrm{CH}_{3} \mathrm{NH}_{3} \mathrm{I}$ 的异丙醇溶液中直至薄膜由黄色完全转变 为黑色, 取出后用异丙醇淋洗, 旋干, 然后在 $70{ }^{\circ} \mathrm{C}$ 下 退火 $30 \mathrm{~min}$.

图 $3 \mathrm{a}, 3 \mathrm{~d}$ 为较致密 $\mathrm{PbI}_{2}$ 薄膜 (图 2a) 直接浸入 $\mathrm{CH}_{3} \mathrm{NH}_{3} \mathrm{I}$ 的异丙醇溶液与 $\mathrm{CH}_{3} \mathrm{NH}_{3} \mathrm{I}$ 反应获得 $\mathrm{CH}_{3} \mathrm{NH}_{3} \mathrm{PbI}_{3}$ 层(方法 $\mathrm{a}$ ); 图 3b, 3e 为多孔 $\mathrm{PbI}_{2}$ 薄膜(图 2b) 直接浸入 $\mathrm{CH}_{3} \mathrm{NH}_{3} \mathrm{I}$ 的异丙醇溶液与 $\mathrm{CH}_{3} \mathrm{NH}_{3} \mathrm{I}$ 反应获得 $\mathrm{CH}_{3} \mathrm{NH}_{3} \mathrm{PbI}_{3}$ 层(方法 b); 图 3c, 3f 为多孔 $\mathrm{PbI}_{2}$ 薄膜(图 2b) 经过异丙醇润洗后浸入 $\mathrm{CH}_{3} \mathrm{NH}_{3} \mathrm{I}$ 的异丙醇溶液与 $\mathrm{CH}_{3} \mathrm{NH}_{3} \mathrm{I}$ 反应获得 $\mathrm{CH}_{3} \mathrm{NH}_{3} \mathrm{PbI}_{3}$ 层(方法 $\mathrm{c}$ ). 三种方法获 得的 $\mathrm{CH}_{3} \mathrm{NH}_{3} \mathrm{PbI}_{3}$ 层颗粒大小依次增大, 分别为 150,250 和 $350 \mathrm{~nm}$, 并且随着颗粒尺寸的增加 $\mathrm{CH}_{3} \mathrm{NH}_{3} \mathrm{PbI}_{3}$ 覆盖 层产生孔洞结构, 使得部分 $\mathrm{TiO}_{2}$ 骨架层裸露出来. 从吸 收光谱(图 4)上可以看出三种方法获得的 $\mathrm{CH}_{3} \mathrm{NH}_{3} \mathrm{PbI}_{3}$ 层 的光吸收强度依次增强, 薄膜的颜色也由棕到黑依次加 深. (a)方法制备 $\mathrm{CH}_{3} \mathrm{NH}_{3} \mathrm{PbI}_{3}$ 层由于 $\mathrm{PbI}_{2}$ 薄膜较为致密, 直接浸入 $\mathrm{CH}_{3} \mathrm{NH}_{3} \mathrm{I}$ 的异丙醇溶液中与 $\mathrm{CH}_{3} \mathrm{NH}_{3} \mathrm{I}$ 迅速反 应使得表面 $\mathrm{PbI}_{2}$ 迅速转变为颗粒较小 $\mathrm{CH}_{3} \mathrm{NH}_{3} \mathrm{PbI}_{3}$ 层, 阻止了内部 $\mathrm{PbI}_{2}$ 的进一步转换, 导致反应不完全, 引起 $600 \sim 800 \mathrm{~nm}$ 范围可见光吸收较弱. (b)方法制备 $\mathrm{CH}_{3} \mathrm{NH}_{3} \mathrm{PbI}_{3}$ 层由于 $\mathrm{PbI}_{2}$ 膜的多孔结构使得 $\mathrm{CH}_{3} \mathrm{NH}_{3} \mathrm{I}$ 溶 液可以渗入孔洞中 $\mathrm{PbI}_{2}$ 的反应较(a)完全, 吸收增强. (c) 方法制备 $\mathrm{CH}_{3} \mathrm{NH}_{3} \mathrm{PbI}_{3}$ 层, 经过润洗后在 $\mathrm{PbI}_{2}$ 上残留一 层异丙醇液膜, 反应过程受到离子扩散影响, 导致反应 界面反应物浓度降低, 使得生成的 $\mathrm{CH}_{3} \mathrm{NH}_{3} \mathrm{PbI}_{3}$ 颗粒粒 径增加, 吸收光谱的增强部分原因是由于颗粒大小增加 引起的散射作用增强所致 ${ }^{[11]}$. 图 5 为 $\mathrm{PbI}_{2}$ 和 $\mathrm{CH}_{3} \mathrm{NH}_{3} \mathrm{PbI}_{3}$ 光活性层的 $\mathrm{X}$ 射线衍射(XRD) 图谱. 从 $\mathrm{a}, \mathrm{b}$ 和 $\mathrm{c}$ 中可以看出三种 $\mathrm{CH}_{3} \mathrm{NH}_{3} \mathrm{PbI}_{3}$ 光活性层衍射谱中存 在明显的 $\mathrm{CH}_{3} \mathrm{NH}_{3} \mathrm{PbI}_{3}$ 和 $\mathrm{PbI}_{2}$ 的衍射峰, 表明三种方法 制备的 $\mathrm{CH}_{3} \mathrm{NH}_{3} \mathrm{PbI}_{3}$ 层中均存在 $\mathrm{PbI}_{2}$ 的残留, 研究表明 适当的 $\mathrm{PbI}_{2}$ 残留可以减少电子复合提高电池的光电转 换效率 ${ }^{[18]}$.

\section{4 不同 $\mathrm{CH}_{3} \mathrm{NH}_{3} \mathrm{Pbl}_{3}$ 薄膜对太阳电池性能的影响}

图 6 给出了三种不同工艺制备有机/无机钲钛矿电 池的 $J-V$ 曲线. 电池的详细性能参数在表 1 中给出. (a),

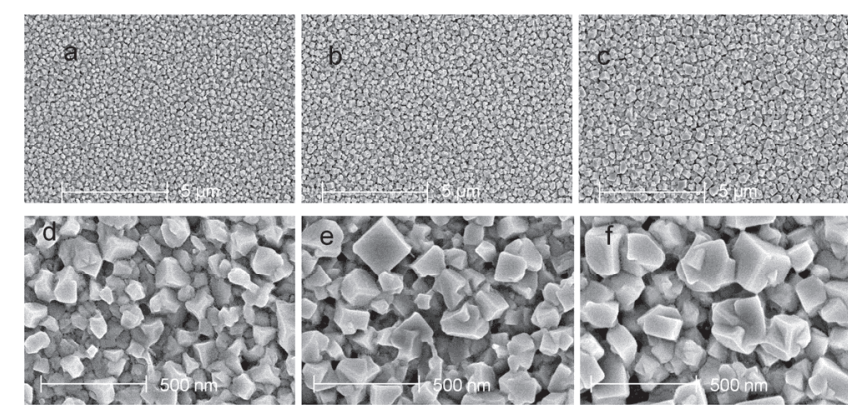

图 3 三种不同工艺制备的 $\mathrm{CH}_{3} \mathrm{NH}_{3} \mathrm{PbI}_{3}$ 光活性层的 $\mathrm{SEM}$ 图. a, d 方法 (a)制备; b, e 方法(b)制备; c, f 方法(c)制备

Figure 3 SEM images of $\mathrm{CH}_{3} \mathrm{NH}_{3} \mathrm{PbI}_{3}$ with different fabrication methods. a, d were fabricated with process (a); b, e were fabricated with process (b); c, f were fabricated with process (c)

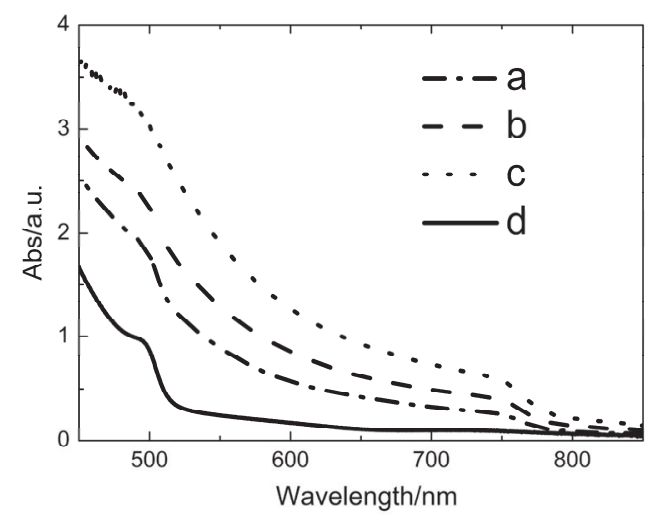

图 $4 \mathrm{PbI}_{2}$ 和 $\mathrm{CH}_{3} \mathrm{NH}_{3} \mathrm{PbI}_{3}$ 光活性层的吸收光谱. a (a)方法制备 $\mathrm{CH}_{3} \mathrm{NH}_{3} \mathrm{PbI}_{3}$ 层; $\mathrm{b}$ (b) 方法制备 $\mathrm{CH}_{3} \mathrm{NH}_{3} \mathrm{PbI}_{3}$ 层; c (c) 方法制备 $\mathrm{CH}_{3} \mathrm{NH}_{3} \mathrm{PbI}_{3}$ 层; $\mathrm{d} \mathrm{PbI}$ 薄膜的吸收光谱

Figure 4 Absorption spectrum of $\mathrm{PbI}_{2}$ and $\mathrm{CH}_{3} \mathrm{NH}_{3} \mathrm{PbI}_{3}$ films. a, b and c were the absorption spectrum of $\mathrm{CH}_{3} \mathrm{NH}_{3} \mathrm{PbI}_{3}$ films: $\mathrm{CH}_{3} \mathrm{NH}_{3} \mathrm{PbI}_{3}$ which was fabricated through process (a), (b) and (c), respectively; d: absorption spectra of the $\mathrm{PbI}_{2}$ film

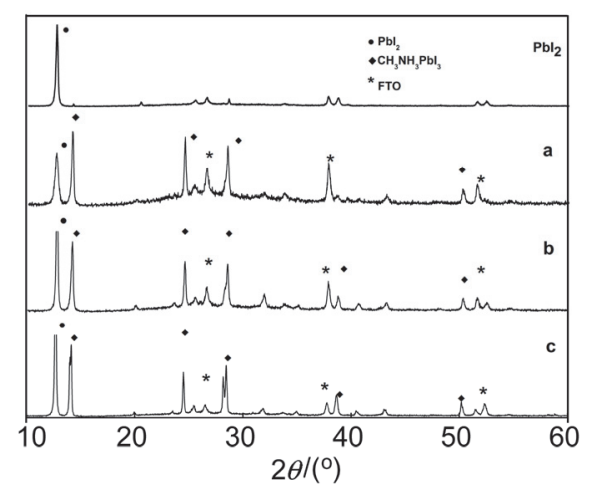

图 $5 \mathrm{PbI}_{2}$ 和 $\mathrm{CH}_{3} \mathrm{NH}_{3} \mathrm{PbI}_{3}$ 光活性层的 $\mathrm{X}$ 射线衍射(XRD)图谱. $\mathrm{a}, \mathrm{b}$ 和 $\mathrm{c}$ 分别为采用 (a), (b) 和(c)三种不同方法制备的 $\mathrm{CH}_{3} \mathrm{NH}_{3} \mathrm{PbI}_{3}$ 光活性层的 $X$ 射线衍射(XRD)图谱

Figure 5 XRD patterns of $\mathrm{PbI}_{2}$ and $\mathrm{CH}_{3} \mathrm{NH}_{3} \mathrm{PbI}_{3}$ with different fabrication methods. a, b and c were fabricated with process (a), (b) and (c) respectively 
(b) 和 (c) 三种方法制备的电池光电转换效率分别为 $10.1 \% ， 13.5 \%$ 和 $10.0 \%$. 其中采用(b)方法制备的太阳电 池性能最高, 其中短路电流密度为 $17.8 \mathrm{~mA} / \mathrm{cm}^{2}$, 开路 电压达到 $1.05 \mathrm{~V}$, 填充因子为 $72.5 \%$. (a)方法制备的太 阳电池由于 $\mathrm{PbI}_{2}$ 的转化不完全引起吸收较低导致电池 的短路电流较小, 仅为 $13.9 \mathrm{~mA} / \mathrm{cm}^{2}$. 而利用(c)方法制 备的太阳电池, 开路电压和填充因子相比(a), (b)两种方 法明显较小, 其中开路电压仅为 $0.90 \mathrm{~V}$. 部分原因是由 于生成 $\mathrm{CH}_{3} \mathrm{NH}_{3} \mathrm{PbI}_{3}$ 颗粒较大造成部分 $\mathrm{TiO}_{2}$ 层裸露, 引 起电池复合增加.

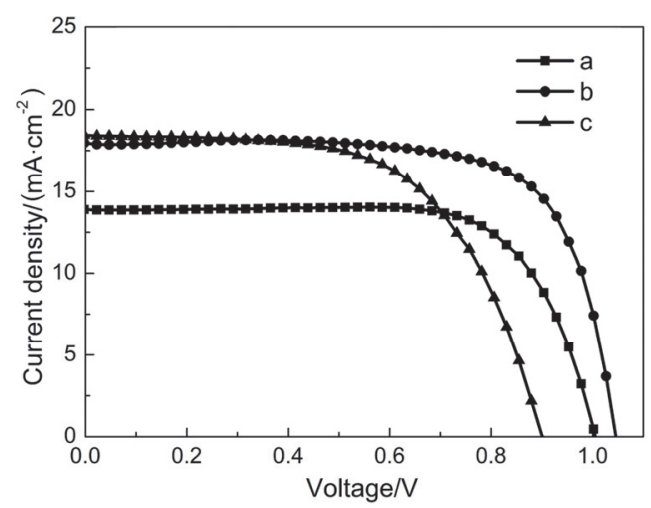

图 6 三种不同工艺制备的有机/无机钘钛矿电池的 $J-V$ 曲线. $\mathrm{a}, \mathrm{b}$ 和 $\mathrm{c}$ 分别为采用(a), (b)和(c)三种不同方法制备的钙钣矿电池的 $J-V$ 曲线. 测试光强为 $100 \mathrm{~mW} \cdot \mathrm{cm}^{-2}, \mathrm{AM} 1.5$, 电池有效面积 $0.09 \mathrm{~cm}^{2}$

Figure $6 J-V$ curves of devices with different fabrication methods. a, b and $\mathrm{c}$ were fabricated with process (a), (b) and (c) respectively. Tested under $100 \mathrm{~mW} \cdot \mathrm{cm}^{-2}$, AM 1.5 , effective area $=0.09 \mathrm{~cm}^{2}$

表 1 不同制备工艺太阳电池的光电性能参数

Table 1 Photovoltaic parameters of devices with different fabrication methods

\begin{tabular}{lllll}
\hline 方法 & $J_{\mathrm{sc}}\left(\mathrm{mA} \cdot \mathrm{cm}^{-2}\right)$ & $V_{\mathrm{oc}} / \mathrm{V}$ & $F F / \%$ & $\mathrm{PCE} / \%$ \\
\hline $\mathrm{a}$ & 13.9 & 1.01 & 71.9 & 10.1 \\
$\mathrm{~b}$ & 17.9 & 1.05 & 72.5 & 13.5 \\
$\mathrm{c}$ & 18.4 & 0.90 & 60.4 & 10.0 \\
\hline
\end{tabular}

$\mathrm{a}, \mathrm{b}$ and $\mathrm{c}$ were fabricated with process (a), (b) and (c) respectively. Tested under $100 \mathrm{~mW} \cdot \mathrm{cm}^{-2}$, AM 1.5 , effective area $=0.09 \mathrm{~cm}^{2}$.

\section{3 结论}

本文研究了不同制备工艺对钙有机/无机钙钛矿太 阳电池钙钛矿层形貌的影响以及对太阳电池的性能影 响. 发现在 $\mathrm{PbI}_{2}$ 制备过程中晾干过程有助于连续沉积法 过程中 $\mathrm{PbI}_{2}$ 的转化, 增加光吸收. 致密的 $\mathrm{PbI}_{2}$ 层造成 $\mathrm{PbI}_{2}$ 的转化不完全, 导致 $\mathrm{CH}_{3} \mathrm{NH}_{3} \mathrm{PbI}_{3}$ 颗粒较小吸收较 弱影响电池的短路电流. $\mathrm{CH}_{3} \mathrm{NH}_{3} \mathrm{PbI}_{3}$ 颗粒过大会引起 $\mathrm{CH}_{3} \mathrm{NH}_{3} \mathrm{PbI}_{3}$ 薄膜孔洞产生造成电池的开路电压下降. $\mathrm{CH}_{3} \mathrm{NH}_{3} \mathrm{PbI}_{3}$ 颗粒的大小为 $250 \mathrm{~nm}$ 的钙钛矿电池获得了 最高的光电转换效率, 达到 $13.5 \%$. 通过对反应条件的 进一步改进有望提高电池的光电转换效率.

\section{4 实验部分}

使用锌粉和一定浓度的盐酸将 FTO 导电玻璃按照 特定图案刻蚀, 洗净, 干燥后 $510{ }^{\circ} \mathrm{C}$ 退火 $30 \mathrm{~min}$. 将电 池的电极部分用胶带保护, 旋涂 $\mathrm{TiO}_{2}$ 致密层前驱体溶 液, 前驱体溶液按照文献方法制备, 用异丙醇稀释至适 当浓度后使用 ${ }^{[19]}$. 致密层溶液采用 $3000 \mathrm{r} / \mathrm{min}$ 旋转 $30 \mathrm{~s}$, 后在 $510{ }^{\circ} \mathrm{C}$ 下退火 $30 \mathrm{~min}$. $\mathrm{TiO}_{2}$ 骨架层制备: 将 $\mathrm{TiO}_{2}$ 浆 料与乙醇按照 $1: 3.5$ (质量比)的比例稀释后超声、摚拌 使浆料分散均匀. 然后将稀释的浆料旋涂在致密层上 (电极同样用胶带保护), 再在 $510{ }^{\circ} \mathrm{C}$ 下烧结 $30 \mathrm{~min}$.

$\mathrm{PbI}_{2}$ 溶液的配置: 称量一定质量的 $\mathrm{PbI}_{2}$ 溶于 $N, N-$ 二 甲基甲酰胺(DMF)中配成 $461 \mathrm{mg} / \mathrm{mL}$ 的 $\mathrm{PbI}_{2}$ 溶液, 然后 在 $70{ }^{\circ} \mathrm{C}$ 下加热搅拌至溶液澄清.

$\mathrm{CH}_{3} \mathrm{NH}_{3} \mathrm{I}$ 的异丙醇溶液的配置: $\mathrm{CH}_{3} \mathrm{NH}_{3} \mathrm{I}$ 按照文献 中的方法合成 ${ }^{[20]}$. 称量 $0.1 \mathrm{~g} \mathrm{CH}_{3} \mathrm{NH}_{3} \mathrm{I}$ 加入到 $10 \mathrm{~mL}$ 异 丙醇溶液中, 搅拌、超声至溶液澄清, 放置备用.

Spiro-OMeTAD 溶液的配置: 将双三氟甲磺酰亚胺 锂溶解在乙腈中配置成浓度为 $520 \mathrm{mg} / \mathrm{mL}$ 的溶液. 将 Co 盐(三[2-(1H-吡唑-1-基)-4-叔丁基吡啶]-钴(III)三(双 (三氟甲基磺酰)亚胺)) KF209)溶解在乙腈中配制成浓度 为 $300 \mathrm{mg} / \mathrm{mL}$ 的溶液. Spiro-OMeTAD 溶液组分: 每毫

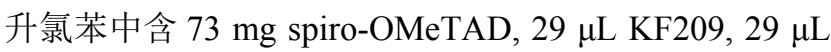
tBP 和 $17.5 \mu \mathrm{L}$ 双三氟甲磺酰亚胺锂的乙腈溶液(520 $\mathrm{mg} / \mathrm{mL})^{[21]}$.

$\mathrm{CH}_{3} \mathrm{NH}_{3} \mathrm{PbI}_{3}$ 光活性层的制备. (a) 将 $\mathrm{PbI}_{2}$ 溶液旋涂在 $\mathrm{TiO}_{2}$ 薄膜上(转速 $3000 \mathrm{r} / \mathrm{min}$, 时长 $30 \mathrm{~s}$ ), 旋涂之后立即 放置在 $70{ }^{\circ} \mathrm{C}$ 的加热台上退火 $30 \mathrm{~min}$. 冷却后浸入 $\mathrm{CH}_{3} \mathrm{NH}_{3} \mathrm{I}$ 的异丙醇溶液中 $20 \mathrm{~s}$, 取出旋干, $70{ }^{\circ} \mathrm{C}$ 下退火 30 min. (b) 将 $\mathrm{PbI}_{2}$ 溶液旋涂在 $\mathrm{TiO}_{2}$ 薄膜上(转速 3000 $\mathrm{r} / \mathrm{min}$, 时长 $30 \mathrm{~s})$, 旋涂之后在室温下晾干, 之后放置在 $70{ }^{\circ} \mathrm{C}$ 的加热台上退火 $30 \mathrm{~min}$. 冷却后浸入 $\mathrm{CH}_{3} \mathrm{NH}_{3} \mathrm{I}$ 的 异丙醇溶液中 $20 \mathrm{~s}$, 取出旋干, $70{ }^{\circ} \mathrm{C}$ 下退火 $30 \mathrm{~min}$. (c) 将 $\mathrm{PbI}_{2}$ 溶液旋涂在 $\mathrm{TiO}_{2}$ 薄膜上(转速 $3000 \mathrm{r} / \mathrm{min}$, 时长 $30 \mathrm{~s}$ ), 室温下晾干, 然后在异丙醇溶液中浸润 $2 \mathrm{~s}$, 接着 浸入 $\mathrm{CH}_{3} \mathrm{NH}_{3} \mathrm{I}$ 的异丙醇溶液中直至薄膜由黄色完全转 变为黑色, 取出后用异丙醇淋洗, 旋干, 然后在 $70{ }^{\circ} \mathrm{C}$ 下退火 $30 \mathrm{~min}$.

接着分别在上述薄膜上旋涂 spiro-OMeTAD 的氯苯 溶液(转速 $4000 \mathrm{r} / \mathrm{min}, 30 \mathrm{~s}$ ), 最后在其上面蒸镀一层 100 $\mathrm{nm}$ 金电极, 即制得 $\mathrm{TiO}_{2} / \mathrm{CH}_{3} \mathrm{NH}_{3} \mathrm{PbI}_{3} /$ spiro-OMeTAD/ $\mathrm{Au}$ 太阳电池. 电池的结构如图 1 所示, 制备好的杂化太 阳电池的有效面积为 $0.09 \mathrm{~cm}^{2}$.

\section{References}

[1] (a) Lee, M. M.; Teuscher, J.; Miyasaka, T.; Murakami, T. N.; Snaith, H. J. Science 2012, 338, 643; (b) Gratzel, M. Nat. Mater. 2014, 13, 838.

[2] (a) Kojima, A.; Teshima, K.; Shirai, Y.; Miyasaka, T. J. Am. Chem. Soc. 2009, 131, 6050; (b) Wu, C. G.; Chiang, C. H.; Tseng, Z. L. J. 
Mater. Chem. A 2014, 2, 15897; (c) Qin, P.; Paek, S.; Dar, M. I.; Pellet, N.; Ko, J.; Gratzel, M.; Nazeeruddin, M. K. J. Am. Chem. Soc. 2014, 136, 8516; (d) Cai, B.; Xing, Y.; Yang, Z.; Zhang, W. H.; Qiu, J. Energy Environ. Sci. 2013, 6, 1480.

[3] Jeon, N. J.; Noh, J. H.; Kim, Y. C.; Yang, W. S.; Ryu, S.; Seok, S. I. Nat. Mater. 2014, 13, 897.

[4] Snaith, H. J. J. Phys. Chem. Lett. 2013, 4, 3623

[5] (a) Yang, Z. S.; Yang, L. G.; Wu, G.; Wang, M.; Chen, H. Z. Acta Chim. Sinica 2011, 69, 627 (杨志胜, 杨立功, 吴刚, 汪茫, 陈红 征, 化学学报, 2011, 69, 627); (b) Yang, Z. S.; Yang, L. G.; Wu, G.; Wang, M.; Tang, B. Z.; Chen, H. Z. Acta Chim. Sinica 2008, 66, 1611 (杨志胜, 杨立功, 吴刚, 汪茫, 唐本忠, 陈红征, 化学学报, 2008, 66, 1611).

[6] (a) Malinkiewicz, O.; Yella, A.; Lee, Y. H.; Espallargas, G. M.; Graetzel, M.; Nazeeruddin, M. K.; Bolink, H. J. Nat. Photonics 2014, 8, 128; (b) Etgar, L.; Gao, P.; Xue, Z.; Peng, Q.; Chandiran, A. K.; Liu, B.; Nazeeruddin, M. K.; Graetzel, M. J. Am. Chem. Soc. 2012, 134, 17396

[7] (a) Yin, W. J.; Shi, T.; Yan, Y. Adv. Mater. 2014, 26, 4653; (b) Stranks, S. D.; Eperon, G. E.; Grancini, G.; Menelaou, C.; Alcocer, M. J.; Leijtens, T.; Herz, L. M.; Petrozza, A.; Snaith, H. J. Science 2013, 342, 341; (c) Xing, G.; Mathews, N.; Sun, S.; Lim, S. S.; Lam, Y. M.; Gratzel, M.; Mhaisalkar, S.; Sum, T. C. Science 2013, 342, 344.

[8] Sun, S.; Salim, T.; Mathews, N.; Duchamp, M.; Boothroyd, C.; Xing, G.; Sum, T. C.; Lam, Y. M. Energy Environ. Sci. 2014, 7, 399.

[9] (a) Kim, H. S.; Lee, C.-R.; Im, J. H.; Lee, K. B.; Moehl, T.; Marchioro, A.; Moon, S. J.; Humphry Baker, R.; Yum, J. H.; Moser, J. E.; Graetzel, M.; Park, N. G. Sci. Rep. 2012, 2, 591; (b) Chen, Q.; Zhou, H.; Hong, Z.; Luo, S.; Duan, H. S.; Wang, H. H.; Liu, Y.; Li, G.; Yang, Y. J. Am. Chem. Soc. 2014, 136, 622; (c) He, M.; Zheng, D.; Wang, M.; Lin, C.; Lin, Z. J. Mater. Chem. A 2014, 2, 5994.

[10] Burschka, J.; Pellet, N.; Moon, S. J.; Humphry Baker, R.; Gao, P.; Nazeeruddin, M. K.; Graetzel, M. Nature 2013, 499, 316.

[11] Im, J. H.; Jang, I. H.; Pellet, N.; Grätzel, M.; Park, N. G. Nat. Nano 2014, 9, 927 .
[12] Xiao, M.; Huang, F.; Huang, W.; Dkhissi, Y.; Zhu, Y.; Etheridge, J.; Gray Weale, A.; Bach, U.; Cheng, Y. B.; Spiccia, L. Angew. Chem., Int. Ed. 2014, 126, 1 .

[13] (a) Wojciechowski, K.; Saliba, M.; Leijtens, T.; Abate, A.; Snaith, H. J. Energy Environ. Sci. 2014, 7, 1142; (b) Wang, J. T.; Ball, J. M.; Barea, E. M.; Abate, A.; Alexander Webber, J. A.; Huang, J.; Saliba, M.; Mora-Sero, I.; Bisquert, J.; Snaith, H. J.; Nicholas, R. J. Nano Lett. 2013, 14, 724.

[14] (a) Zhu, Z.; Bai, Y.; Zhang, T.; Liu, Z.; Long, X.; Wei, Z.; Wang, Z.; Zhang, L.; Wang, J.; Yan, F.; Yang, S. Angew. Chem., Int. Ed. 2014, 126, 1; (b) Wang, K. C.; Jeng, J. Y.; Shen, P. S.; Chang, Y. C.; Diau, E. W.; Tsai, C. H.; Chao, T. Y.; Hsu, H. C.; Lin, P. Y.; Chen, P.; Guo, T. F.; Wen, T. C. Sci. Rep. 2014, 4, 4756; (c) Wang, K. C.; Shen, P. S.; Li, M. H.; Chen, S.; Lin, M. W.; Chen, P.; Guo, T. F. ACS Appl. Mater. Interfaces 2014, 6, 11851.

[15] (a) Wang, Q.; Dong, Q.; Xiao, Z.; Yuan, Y.; Huang, J. Energy Environ. Sci. 2014, 7, 2359; (b) Seo, J.; Park, S.; Chan Kim, Y.; Jeon, N. J.; Noh, J. H.; Yoon, S. C.; Seok, S. I. Energy Environ. Sci. 2014, 7, 2642; (c) Liu, D.; Kelly, T. L. Nat. Photonics 2013, 8, 133; (d) Ryu, S.; Noh, J. H.; Jeon, N. J.; Kim, Y. C.; Yang, S.; Seo, J. W.; Seok, S. I. Energy Environ. Sci. 2014, 7, 2614.

[16] Liu, M.; Johnston, M. B.; Snaith, H. J. Nature 2013, 501, 395.

[17] Shi, J.; Luo, Y.; Wei, H.; Luo, J.; Dong, J.; Lv, S.; Xiao, J.; Xu, Y.; Zhu, L.; Xu, X.; Wu, H.; Li, D.; Meng, Q. ACS Appl. Mater. Interfaces 2014, 6, 9711.

[18] Chen, Q.; Zhou, H.; Song, T. B.; Luo, S.; Hong, Z.; Duan, H. S.; Dou, L.; Liu, Y.; Yang, Y. Nano Lett. 2014, 14, 4158.

[19] Kim, J. Y.; Kim, S. H.; Lee, H. H.; Lee, K.; Ma, W.; Gong, X.; Heeger, A. J. Adv. Mater. 2006, 18, 572.

[20] Baikie, T.; Fang, Y.; Kadro, J. M.; Schreyer, M.; Wei, F.; Mhaisalkar, S. G.; Graetzel, M.; White, T. J. J. Mater. Chem. A 2013, 1,5628 .

[21] Jeon, N. J.; Lee, J.; Noh, J. H.; Nazeeruddin, M. K.; Gratzel, M.; Seok, S. I. J. Am. Chem. Soc. 2013, 135, 19087.

$(\mathrm{Lu}, \mathrm{Y}$. 